\title{
Oral FimH inhibitors effective against UTI
}

A team of researchers from Washington University School of Medicine have developed a promising preclinical candidate for the oral treatment of UTI. Mannosides that specifically inhibit the FimH type 1 pilus lectin of uropathogenic E. coli (UPEC) - the causative agent for more than $85 \%$ of all UTIs-have demonstrated therapeutic potential for the oral treatment and prophylaxis of UTIs in mouse studies.

\section{4 ...able to treat an established UTI by oral administration with just a single dose 77}

These novel compounds were able to clear chronic cystitis within $6 \mathrm{~h}$ of treatment, as indicated by a 3-log drop in bacterial titer. "The new mannosides represent the most potent inhibitors of FimH/UTI ever reported-able to treat an established UTI by oral administration with just a single dose," says lead researcher Scott Hultgren.

Resistance of UPEC to the standardof-care antibiotic, trimethoprimsulfamethoxaxole (TMP-SMZ), has risen dramatically in recent years and led to high recurrence rates. "Millions of women suffer from chronic or recurrent UTI and often require long-term prophylactic antibiotic therapy - a regimen that is becoming progressively less effective with the rise in antibiotic resistance," Hultgren adds.
Importantly, in addition to effectively treating existing infection, these mannosides also potentiated the activity of TMP-SMZ and prevented subsequent infection when given prophylatically. If these early mouse studies are clinically translatable into humans, these drugs have the potential to increase the efficacy of TMP-SMZ and shorten treatment courses, resulting in fewer treatment failures and reduced antibiotic resistance. "[These compounds] ensure the clearance of infection by subverting the UPEC virulence factor necessary for persistence-even in the face of TMP-SMZ resistance," explains Hultgren.

Currently, the team are investigating techniques to improve the oral bioavailability of mannosides and enhance penetration into bladder tissue. "We are following medicinal chemistry strategies to increase the $\log \mathrm{D}$, $\mathrm{pKa}$, and half-life of these compounds," says Hultgren. The team's ultimate goal is to create compounds that can completely eliminate intracellular bacterial communities (IBCs) and reservoirs of tissue-associated bacteria. "IBCs are a primary reason why we are optimizing the compounds for increased tissue penetration," adds Bradley Ford, a fellow researcher within the team. "UTI is not just an infection of the urine. Autoinfection by re-emergence of IBCs is a mechanism of chronic UPEC infection."

Pending successful toxicity studies and funding, the group predicts that human clinical trials could commence in less

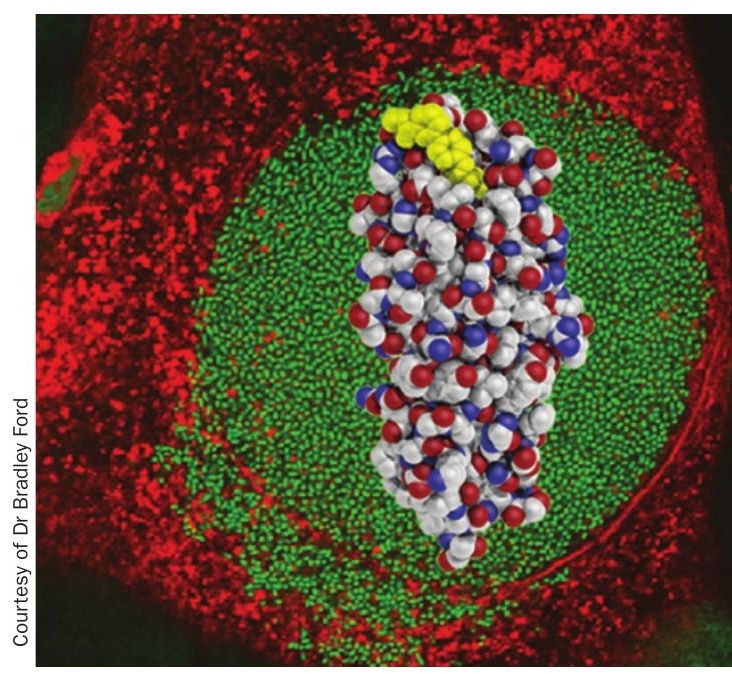

than two years. "A clinical trial might comprise frequent recurrent or chronically infected $E$. coli UTI patients in whom episodic acute drug therapy has failed," Hultgren suggests. "Patients would receive a standard course of TMP-SMZ, plus mannoside or placebo."

For catheter-associated UTIs, an alternative approach might be to line catheters with these FimH inhibitors. "We are currently developing models to investigate the applicability of this approach," Hultgren reveals.

Melanie Clyne

Original article Cusumano, C. K. et al. Treatment and prevention of urinary tract infection with orally active FimH inhibitors. Sci. Transl. Med. doi:10.1126/ scitranslmed.3003021 